\title{
Die Könige und das Reformationsverfahren des Rechts in den polnischen Städten vom 14. bis zum 16. Jahrhundert
}

\begin{abstract}
Magdeburg law was in force in many Polish cities as a result of location and translocation from Polish to German law. Create and modify rights can take many forms in the Polish cities of the late Middle Ages and Renaissance: legislation of king and parliament, municipal legislation, legal-theoretical proposals and modifications of legal manuscripts (Mirror of Saxony and Weichbild of Magdeburg). According to the Magdeburg law, change of the written law required a consent of the owner of the city, so in the case of the royal cities - of the monarch. Thus, the legislative procedure in many cases require the mandatory participation of the king: in cases of a new regulation was contrary to the written law (criminal law, civil law, the city system and other issues addressed in the Mirror of Saxon and Weichbild of Magdeburg) and when he played a role as a legislator in the king's powers (taxes, duty exemptions, business licenses, et al.). Most of the regulations was initiated by stakeholders (municipalities, citizens et al.). The process of the reformation of the municipal law includes not only the replacement of the existing regulations and adding the new regulations, but also the necessary steps preceding and following the legislation sensu stricto (E.g. the cities regularly gave "gifts" to public officials, hoping for their support).
\end{abstract}

\section{1.}

Die Rezeption des Sächsisch-Magdeburger Rechts im Königreich Polen beginnt in der ersten Hälfte des 13. Jahrhunderts ${ }^{1}$. Die ausländischen Siedler in Polen wandten dieses Recht an. Jedoch haben die polnischen Einwohner noch lange das polnische Recht

${ }^{1}$ Die Forschungstand über das SächsischMagdeburgischen Rechts in Polen wurde beschrieben in: GÖNCZI, Geschichtlicher Überblick 19-32; WIELAND, GÖNCZI, Forschungsüberblick 39-67; JANICKA, Wkład polskich historyków 47-75. Über die Verbreitung des Magdeburger Rechts siehe besonders: KAMIŃSKA, Lokacje 71; ŁYSIAK, Ius supremum; LÜCK, Sachsenspiegel und Magdeburger Recht; JANICKA, Die Rezeption 61-74; LIEBERWIRTH, Einführung oder Rezeption 167-180; ZAJDA, Deutsche Einflüsse 289-304; LÜCK, Über den Sachsenspiegel; EBEL, Rechtsentstehung und Rechtstransfer 37-47; LÜCK, Sachsenspiegel und Magdeburger Recht. Grundlagen 100-104; dort frühere Literatur. angewandt, obwohl das deutsche Recht bereits in ihren Orten offiziell in Kraft gesetzt worden war. Die Ergebnisse der Forschungen, die nach dem zweiten Weltkrieg geführt wurden, zeigen, dass das polnische Privatrecht und Strafrecht in den Dörfern noch bis Ende des 15. Jahrhunderts angewandt wurde. ${ }^{2}$ Der Prozess des Magdeburger Rechtstransfers dauerte sehr lange und aus diesem Grund können wir nicht von einer vollen Rezeption reden, sondern von der Adaptation (die selektive Rezeption und die Modifikation der Magdeburger- und Polnischen Regulationen ${ }^{3}$ ).

\footnotetext{
${ }^{2}$ MAtUSZEWSKI, Prawo niemieckie 59-60; ZAJĄCZKOWSKI, Lokacje osad wiejskich 11, 136; SAMSONOWICZ, Kto podejmował decyzje 157.

${ }^{3}$ Vrg. EBEL, Rechtsentstehung und Rechtstransfer 38. Ebel bindet mit dem "Transfer" solche Begriffe: Übertragung, Veränderung, Rezeption. Auf dem Polnischen Grund benutzt man lieber die Begriffe
} 
Im Mittelalter verband man in Polen mit dem Begriff des deutschen Rechts (ius Teutonicum) sehr oft nur drei Elemente: 1. eine bestimmte Liste von Pflichten und Leistungen der Einwohner für einen Grundherrn (eine Garantie für die Freiheiten ${ }^{4}$ ) 2. die Gerichtsjurisdiktion des Grundherrn, 3. Verwaltungsorgane - u.a. Vogt, Schöffen, Rat, Bürgermeister. ${ }^{5}$ Jedoch, verbreitete sich das Sächsisch-Magdeburger Privat- und Strafrecht seit dem 13. Jh. langsam konsequent. Es gab immer mehr Handschriften des so gennannten primären Rechts - Speculum Saxonum und Magdeburger Weichbildrecht - in lateinischen Übersetzungen von Konrad von Oppeln und Konrad von Sandomierz ${ }^{6}$. Eine große Rolle spielten auch die Urteile von Magdeburg, Halle, Posen und Krakau ${ }^{7}$. Das Magdeburger Recht musste jedoch, je mehr es angewandt wurde, umso mehr modifiziert werden, um es den lokalen Verhältnissen anzupassen. Aus der ersten Hälfte des 14. Jhs. stammen die ersten Rechtsreformationen für Kleinpolen aus Krakau [Kraków]. Sie umfassten das Erbrecht, Eherecht und Strafrecht. Schon

„Rezeption“ und „Einflüsse“ statt „Transfer". Vrg. : BARDACH, Recepcja 1-26.

${ }^{4}$ LIEBERWIRTH, Einführung oder Rezeption 169; EBEL, Rechtsentstehung und Rechtstransfer 40-42.

${ }^{5}$ Vgl. MATUSZEWSKI, Prawo sądowe 52-59; MATUSZEWSKI, Rodzaje własności gruntu 158, 160-162, 164.

${ }^{6}$ Siehe: HALBAN, Zur Geschichte des deutschen Rechtes 95-101; LIEBERWIRTH, Einführung oder Rezeption 171, 173-174; CARLS, Rechtsquellen 73-98, auch Stichworte im Repertorium „Geschichtsquellen des deutschen Mittelalters": Sachsenspiegel; Weichbildrecht, Das Sächsische; Ius municipale Magdeburgense Versio Sandomiriensis; Ius municipale Magdeburgense Versio Nicolai Iaskier [www.geschichtsquellen.de] (abgerufen am:

29. 10. 2013). Verzeichnis der Handschriften, die in Polen angewandt wurden, siehe: OPITZ, Deutsche Rechtsbücher 21-32, 46-48. Die kritische Edition der Handschriften Nr. 170a und 170b aus dem Jagiellonen Bibliothek in Krakau: PIIRAInen, WAssen (Hg.), Der Sachsenspiegel. Das Vergleich der lateinischen Handschriften des Sachsenspiegels siehe: RYMASZEWSKI, Łacińskie teksty.

${ }^{7}$ CARLS, Rechtsquellen 99-103. Dort die Verzeichnis der zahlreichenden Editionen. früher waren die Beziehungen zwischen den Stadtorganen anders als in Magdeburg organisiert, insbesondere wurden die Funktionen des Stadtrates immer umfangreicher. Diese Reformen wurden von Kazimierz dem Großen in den Jahren 1336, 1342, 1358 und 1363 bestätigt. $^{8}$

Es wäre wissenswert, wie sich das Recht in den polnischen Städten entwickelt hat. Die Bedeutsamkeit dieses Themas hat Barłomiej Groicki schon vor 450 Jahren betont. Er schrieb im Jahre 1559, dass das deutsche Recht in den polnischen Städten nicht mehr angewandt wurde, sondern das polnische Stadtrecht. ${ }^{9}$ Was ist also zwischen 1336 (Privilegium für Krakau) und 1559 (erste Auflage von Porzadek sąów von Bartłomiej Groicki - Stadtgerichts- und Prozessordnung des Magdeburger Rechts im Kronland Polen) passiert, dass Groicki von einem polnischen Recht sprechen konnte?

\section{2.}

Die Rechtsquellen in den königlichen Städten Polens lassen sich in sechs Gruppen einteilen. Der Ausgangspunkt war natürlich das primäre Recht (der Sachsenspiegel, das Weichbildrecht und die Magdeburger Urteile), das modifiziert wurde: 1.) von der Gesetzgebung des Königs und des Parlaments; 2.) von der Gesetzgebung der Stadtorgane (Willkür und Lauda); 3.) von den Modifikationen in den Handschriften des Sachsenspielgels und Weichbilds, die von den Kopisten gemacht wurden; 4.) von den Vorschlägen der gelehrten Juristen; 5.) von den Präzedenzen der oberen Gerichtshöfe (unter anderen in Krakau [Kraków], Posen [Poznań], Lemberg [Lwów, Lviv] und Kalisz). Die wichtigste Regel der Stadtgesetzgebung wurde in der Glosse zum Weichbildrecht ausgedrückt:

\footnotetext{
${ }^{8}$ Siehe STARZYŃSKI, Krakowska rada miejska, 100-11ß dort die Analyse der früherer Werken, besonders von Patkaniowski, Estreicher, Grodecki und Wyrozumski. GROICKI, Porządek sądów 6.

[http://www.wbc.poznan.pl] (abgerufen am: 31. 12. 2012).
} 
der Stadtrat durfte nur Vorschriften einführen, die mit dem schriftlichen Recht übereinstimmend waren. ${ }^{10}$ Deshalb waren nicht alle vorhin genannten Modifikationen legal besonders Veränderungen der Handschriften durch Kopisten. Wenn der Rat (in den königlichen Städten) das schriftliche Recht reformieren wollte, brauchte er die Zustimmung des Königs, der Hauptgesetzgeber und Stadtherr war. Aber sogar der König konnte arbiträr nicht den Sachsenspiegel und das Weichbildrecht abändern. In der Glosse zum Weichbildrecht befindet sich eine Liste von Gründen, aus denen man das schriftliche Recht modifizieren durfte. Sie sind ähnlich wie die Liste der Rechtsmerkmale von Isidorius von Sevilla. ${ }^{11}$

Aus diesem Grund kann man sagen, dass die Reformation des primären Rechts in polnischen königlichen Städten nur von dem König, eventuell von dem Sejm (Parlament) ${ }^{12}$ durchgeführt werden durfte.

In den folgenden Beispielen stelle ich zwei Typen von königlichen Akten vor: Einerseits die Modifikationen des schriftlichen primären Rechts (am Beispiel des Erbrechts) andererseits solche Regulationen, die nur in der Kompetenz des Königs lagen und früher nicht im primären Recht geregelt waren (beispielweise Steuer- und Zollbefreiungen). In diesem Kontext möchte ich betonen, dass in der königlichen Gesetzgebung für die Städte nicht nur die Schaffung eines

\footnotetext{
${ }^{10}$ SzIM Art. 14, Glosse 10; JIM Art. 14, fol. 15. Siehe: GROICKI, Artykuły prawa majdeburskiego 86.

${ }^{11}$ Das sind: die Güte als Rechtsziel, die Richtigkeit, die Anwendbarkeit, die Übereinstimmung mit der Menschennatur, die Übereinstimmung mit den lokalen Sitten, die Adäquatheit zur Zeit und zum Ort, die Notwendigkeit, die Klarheit, der Nutzen, die Suprematie des bonis commune über die partikularen Interessen.

${ }^{12}$ Die Rolle des Sejms als Gesetzgeber wurde seit dem 16. Jh. immer grösser. Die Adeligen strebten danach, die Kontrolle über die königlichen Städten für Sejm zu bewahren. Während der sogenannten Exekution des Sejms in den Jahren 1562-1565 wurden die Gesetze erlassen, die u.a. die Kontrolle über die Stadtfinanzen der Starostei garantierten.
}

Rechtsakts wichtig war, sondern auch auf bestimmte Handlungenhinweisen, die davor und danach erfolgten. Meine Schlussfolgerungen basieren auf Quellen der königlichen Städte, besonders aus den ehemaligen Woiwodschaften von Krakau, Lublin und Sandomierz.

\section{3.}

Seit dem 15. Jh. wurden mehrere königliche Akten in der so genannten Polnischen Metrik (Metryka Koronna) als Kopien registriert. Die Metrik wurde von Kanzlern mit dem Hilfe der Sekretären in Kanzleien geführt. Dort kann man nicht nur die normativen und „ewigen" Akten finden, sondern auch die normativ-temporären Ordinationen und Rechtsverwirklichungsurkunden, welche seit Ende des 15. Jhs. stammen. Viele dieser Schriften betreffen die Städte. Von den Narrationen der Urkunden erfahren wir, dass der König nur selten die Gesetzgebung für eine Stadt initiiert hat - die Bürger übernahmen diese Rolle. In den Urkunden war die Initiative in solchen Formen bezeichnet: "[fol. 48v] Sigismundus Augustus etc. Significamus etc. Exhibitum esse coram nobis nomine proconsulis et consulum, ac totius communitatis civitatis nostre Ilkussiensis plebiscitium de gierada sive paraferna communi consensi inter eos factum. Supplicatumque est nobis eorum nomine, ut scripsum plebiscitum pro commune bone eorum factum, ratum habere, atque [fol. 49r] illud approbare et confirmare authoritate nostra Regia dignaremur13; „[fol. 295r] Confirmatio articulorum oppidanorum Czieskovensis. Sigismundus Augustus etc. Significamus etc. Exhibitos esse coram nobis per certos consiliaros nostros nomine incolarum oppidi nostri Czieskoviensis plebisciti articulos in papyro scriptos et sigillis subappenssis commitentes per proconsulem, consules, viceadvocatum, scabinos, seniores contuberniorum et totam communitatem eiusdem oppidi nostri unanimi omnium consilio,

\footnotetext{
${ }^{13}$ Olkusz: MK 89, fol. 48-49v vom 5. 11. 1556.
} 
voluntate et assenssione editos, et sancitos [...]"14; "[fol. 148v Institucio gieradae in civitate Bidgosthensis. [...] Dei gratia Rex Polonie etc. Manifestum facimus tenore presentium universis et singulis praesentium et futuris harum notitiam habituris, quia cum famati proconsul et consulibus civitatis nostrae Bidgostiensis, suis totiusque communitatis civium et incolarum Bidgostiensis nominibus, ad nos certas personas misissent, humiliter per eos nobis supplicasset [...]."15

Die Forschungen zum Stadtbudget im Mittelalter und in der frühen Neuzeit zeigen, dass die Stadträte Geld für legislative Tätigkeiten reserviert hatten. Die Ausgaben umfassten nicht nur die Kosten der Reisen (die Jagiellonen waren noch reges ambulantes), sondern und vor allem - "Geschenke" für Beamte, besonders Beamte der königlichen Kanzleien. ${ }^{16}$ Der Fall von Krakau zeigt, dass manche Beamte, beispielsweise der palatinus Cracoviensis, ständig bezahlt wurden. Im Austausch haben die Beamten Rechtshilfe und Vermittlung als Gegenleistung garantiert. Nur manchmal finden wir Namen der Vermittler in den Urkunden, besonders wenn der Antragssteller eine kleine Stadt war. ${ }^{17}$ Sehr oft war es der Vizekanzler Piotr Tomicki (1515153518) ein Protektor vor Zygmunt I. (15071548). ${ }^{19}$

\footnotetext{
${ }^{14}$ Mik.R. vom 24. 12. 1550.

${ }^{15}$ Bydgoszcz: MK 100, fol. 148v-149r von 1556.

${ }^{16}$ Siehe: NoGA, Relacje władz 119-135; NoGA, Krakowska rada miejska 98, 88-89, 93-106; STARZYŃSKI, Krakowska rada miejska 133-144.

${ }^{17}$ Beispielsweise die Urkunde für Anna Milanowska aus dem Jahre 1524: Quia nos ad intercessionem magnifici Stanislai Odrowansch de Sprowa palatini Podolie sincere nobis dilecti pro parte nobilis Anne Milanowska apud nos factam eiusdem domum extra muros civitatis nostre Lublinensis in ciniterio monasterii Sancti Bernardini iacentem quam ad praesens possidet ab omnibus censibus et contributionibus nostris et civilibus de certa sciencia et gratia nostra eximendum, absolvendum et libertandum duximus, eximimusque absolvimus, et libertamus, temporibus perpetuis et in eum litteris nostris presentibus." (Mik.B. Nr. 5 vom 24.3.1540).

${ }^{18}$ CHŁAPOWSKI u.a., Urzędnicy centralni i nadworni. Nr. 634, S. 108.

${ }^{19}$ OdRZYWOLSKA-KIDAWA, Prace kancelaryjne 2, 160.
}

Man muss betonen, dass "Geschenke“ nicht immer den Erfolg garantierten; vor allem, wenn die geforderte Regelung alte Privilegien verletzte. Beispielweise wollte der Stadtherr von Góra Jana [Johannesberg, 30 km von Krakau entfernt] einen wöchentlichen Markt organisieren. Dazu brauchte er die königliche Zustimmung in der Form des Privilegs ${ }^{20}$. Obwohl er eine Urkunde bekommen hatte, durfte er diese Erlaubnis nicht ausüben, weil ein Markt $10 \mathrm{~km}$ von Johannesberg in der Stadt Dobczyce schon früher bestand und die Bewohner von Dobczyce die Garantie gegen Konkurrenz hatten. ${ }^{21}$

Auch in anderen Fällen war die königliche Zustimmung leicht erreichbar. Davon zeugen die Formulare der Urkunden für ähnliche Fälle beispielweise für Steuerbefreiungen, die besonders nach Zerstörungen durch Brände beantragt wurden: „[S. 251] Libertas oppidanorum de Polanyecz. Sigismundus etc. significamus tenore presentium universis. Quia nos comparientes calamitati concivium oppidi nostri Polanyecz quam ex novissima conflagracione illius acceperunt, dedimus et concessimus graciose, damnusque et coaredimus quod presentes ipsis civibus dicti oppidi Polanyecz damna per iurendum possis libertatem ab omnibus et singulis censibus, solutionibus, contributionibus et exactionibus civilibus, que Schoss dicantur pro re publica institutis er instituendis, ad octo annorum decursum a data praesentium computandorum. A podvodis vero ad unum annum, et a contributione czoppowe ad unum quartale anni." 22 In ähnlichen Fällen wechselte man im Formular nur die Stadt und die Fristungen.

Das königliche Gesetzgebungsverfahren für die Städte vom Antrag über die Zustimmung zur Urkunde war also nicht kompliziert. Komplizierter war die Durchsetzung der neuen

20 Es geht hier um so genannte administrative Privilegien, die keine normative Akten waren, sondern schafften sie Berechtigungen für die Benefiziaten. Siehe: IHERING, Der Zweck 314.

${ }^{21}$ ZDANEK, Kopiariusz cystersów szczyrzyckich Nr. 8 fol. 19r-20r (S. 41-43) vom 4. 6. 1468; Nr. 11, fol. 29v$31^{\text {r }}$ (S. 62-65) vom 29.9. 1519; ANKr. Dok.Dep. 36 vom 19. 7. 1523.

22 Połaniec, MK 40, S. 251 vom 18. 10. 1526. 
Vorschriften. Es ist selbstverständlich, dass das neue Recht publiziert werden musste. Seit dem 16. Jahrhundert wurden die königlichen Urkunden für die Städte nicht nur mündlich auf dem Marktplatz bekanntgegeben und in Stadtbüchern eingeschrieben, sondern auch in die so genannten Grodbücher (castriensia) beim zuständigen Landkreisamt eingeschrieben. Außerdem hatten sich die Stadteinwohner um Garantien bemüht, damit die neuen Regulationen eingehalten wurden. Dazu hat der König eine Garantieakte ausgestellt. Regulationen ohne Garantieakte waren sehr oft leges imperfectae - nur normative Kommuniqués.

Die Rolle der Garantieakten muss betont werden. Meiner Meinung nach sollten alle königlichen und parlamentarischen Akte in der Jagiellonen Periode (1386-1572) in drei Gruppen geteilt werden ${ }^{23}$. Zu der ersten Gruppe gehören die normativen Regulationen, die neues Recht schufen; zu der zweiten zählen Rechtsverwirklichungsakte (beispielsweise Zustimmung für die Jahrmärkte ${ }^{24}$, Verpfändung der Stadt ${ }^{25}$ usw.). $\mathrm{Zu}$ der dritten Gruppe, den Garantieakten, gehören im Einzelnen: 1.) Exekutivakten, 2.) Interventionsakten, 3) Bestätigungen. Im 15.

\footnotetext{
${ }^{23}$ Als solche Akte verstehe ich nicht die Urkunden, sondern die nachfolgenden Aktivitäten im Bereich der Gesetzgebung, der Administration oder der Rechtsprechung.

${ }^{24}$ Statement der Märkten- und Messengenehmigungen für Städte in Kleinpolen siehe: SAMSONOWICZ, Przemiany osi drożnych 708-710, 715716; SAMSONOWICZ, Handel Lublina 621-625; SAMSONOWICZ, Niektóre cechy charakterystyczne sieci jarmarcznej 9-14; WYROBISZ, Uwagi o kalendarzu jarmarków 27-32; BoGUCKA, Jarmarki w Polsce 17-20.

${ }^{25}$ Beispielweise LUDWIG, Besteuerung und Verpfändung königlicher Städte und die Rezension dieses Buches: MATUSZEWSKI, SZULC Opodatkowanie i polityka zastawu miast królewskich. Das Thema der Verpfändung der königlichen Güter wurde schon mehrfach in der polnischen Historiographie analysiert. Siehe auch das Historisch-geographische Lexikon von Polen im Mittelalter, dort sowohl Regesten der Quellen als auch bibliographische Hinweisen:

http://www.slownik.ihpan.edu.pl/intro.php, abgerufen am: 15. 1. 2013).
}

und im 16. Jh. wurden die Exekutivakte sehr oft als Exekutivklausel schon in der Urkunde niedergeschrieben. ${ }^{26}$ Als Beispiel kann man Exekutivklausel aus dem Steuerprivileg für Anna Milanowska aus Lublin zeigen: „Proinde tibi magnifico Joanni de Thanczin castellano Woinicensi, marsalco curie nostre et capitaneo Lublinensi sincere nobis dilecto et aliisque pro tempore existentibus capitaneis nostris conservent et conservari faciant capitaneis nostris Lublinensibus pro tempore existentibus eorumque vicesgerentibus id manifestum facimus mandantes, ut eandem Annam Milanowska ipsiusque successores domus predicte possesores pro tempore existentes in hac libertate per nos et premissum est concessa conservent et consevari faciat, non permittatque aliquas contribuciones ab ea et ipsius successoribus racione domus sue predicte repetere. Aliisque pro tempore existentes capitanei nostri conservent et conservari faciant, et illo permittant pro gratia nostra non aliter facturi." 27 In diesen Klauseln hatte der König den Beamten unter Androhung einer Strafe befohlen, die neue Regulation einzuhalten. $\mathrm{Zu}$ den Strafen angedrohten gehörten: Geldstrafen, der Verlust der königlichen Gnade (sehr populär ${ }^{28}$ ), der Amtsverlust und Freiheitsstrafen.

\footnotetext{
${ }^{26}$ SUŁKOWSKA-KURAŚ, Dokumenty królewskie 27, 38, 49.

${ }^{27}$ Lublin (für Anna Milanowska): Mik.B. Nr. 5 vom 24. 3. 1540.

${ }^{28}$ Beispiele aus den Jahren 1386-1450: Biecz (ZDM 4, 1088 vom 2. 5. 1390; MBiecz 13); Krakau (KDMK I.1, 112 vom 20.3. 1419); Opoczno (KDM 4, 1187 vom 23. 2. 1420); Poznań (KDW 8, 945 vom 6.9.1422); Lwów (AGZ 4, 75 vom 1425); Słupca (KDW 8, 1016 vom 27. 2. 1425); Lwów (AGZ 5, 37 vom 25.3.1426); Osiek (ZDM 7, 2046 vom 14.3. 1430); Kazimierz (KDW 9, 1090 vom 23. 5. 1426); Kobylin (KDW 9, 1243 vom 5. 8. 1430); Krakau, Kleparz (KDMK I.1, 128 vom 1. 9. 1430); Radymno - Privatstadt (MAML 71 vom 19. 3. 1431); Krakau (ZDM 7, 2068 vom 10.4. 1431); Tyrawa heutzutage Mrzygłód (ZDM 7, 2081 vom 28. 6. 1431); Radom (ZDM 7, 2101 vom 24. 2. 1434); Szydłowiec - Privatstadt (ZDM 2, 490 vom 26. 8. 1436); Krakau, Kazimierz, Kleparz (KDMK I.1, 251 vom 21.5.1439); Radom (ZDM 3, 840 vom 3. 1. 1450); Krzepice (ZDM 3, 850 vom 20.3.1450); Krzepice (ZDM 3, 851 vom 20.3. 1450); Krasnystaw (ZDM 3, 869 aus 1450); Ryczywół (ZDM 6, 1557 vom
} 
Der König hatte Interventionsakte erstellt, wenn das Recht gebrochen wurde. Die Urkunden nannte man entweder mandatum oder littere universales - je nachdem, ob die Urkunden nur an Beamten oder an andere (besonders Stadteinwohner oder Adelige) adressiert waren. Als Beispiel seien die mandata genannt, die die Könige wegen der Verletzungen von Zoll- und Steuerprivilegien erlassen hatten. ${ }^{29}$

Die Konfirmation (Bestätigung) war sehr oft sowohl eine Garantie für die Berechtigten, als auch eine "Erinnerung" für die Beamten und andere an ihre Pflichten ${ }^{30}$. Gute Beispiele dafür sind die Bestätigungen der Jahrmarkt- und Wochenmarktprivilegien ${ }^{31}$ und der Steuerbefrei-

12. 6. 1389). Siehe: RYMASZEWSKI, Król w dawnej Polsce 181.

${ }^{29}$ Zollprivilegienverletzung: Kalisz (KDW 10, 1617 vom 13. 6. 1443 = Prz. Kal., 33); Kleparz (PPiSMK I.1, 500 vom 25.8. 1558); Lelów (KDM 4, 1105 vom 23. 8. 1407); Lwów (AGZ 5, 85 vom 16. 6. 1441); Lwów (AGZ 5, 148 aus 1456); Nowy Sącz (AGZ 9, 29 vom 18. 8. 1427; AGZ 9, 39 vom 25. 2. 1437); Olkusz (ZDM 72163 vom 5. 4. 1434); Opoczno (KDM 4, 1187 vom 23. 2.1420; KDM 4, 1290 vom 28.7.1432); Poznań (KDW 10, 1613 vom 10.6. 1443); Pyzdry (KDW 9, 1320 vom 16.4.1433); Wiślica (MK 30, fol. $138^{\mathrm{r}}-139^{\mathrm{r}}$ vom 12.2.1518). Steuerprivilegienverletzung: Biecz (MK 49, fol. 89v-90r vom 13. 4. 1534); Bochnia (MK 49, fol. 89v-90r vom 13. 4. 1534); Krakau (BCzart., vol. V/45 vom 29. 6. 1572); Olkusz (Łabęcki 2, 25 aus 1498).

${ }^{30}$ Von Funktionen und Merkmalen der Bestätigungen der Privilegien siehe: MOHNHAUPT, Confirmatio privilegiorum 45-63.

${ }^{31}$ Bochnia - die Bestätigung der freien Fleischverkaufsurkunde vom 16. 3. 1405 (Bestätigungen vom 5. 8. 1494 und 31. 1. 1525, MK 39, S. 129-132); Bochnia - die Bestätigung des Jahrmarkts am 24. Oktober (MK 24, fol. 105r vom 10.5. 1509); Grybów - die Bestätigung des Wochenmarkts (KDM 5, Tasche Q, 99 vom 5.11.1488); Kęty - die Bestätigung der Jahrmärkte (Heck 10 vom 4. 8. 1519); Krakau - die Bestätigung der Jahrmärkte (PPiSMK I.1, 204 vom 3.6. 1566); Krzepice - die Bestätigung des Wochenmarkts am Donnerstag (ZDM 3, 850 vom 20. 3. 1450); Lublin - die Bestätigung der Jahrmärkte (MLublin 184 aus 1512); Łuków - die Bestätigung des Wochenmarkts am Donnerstag (MK 49, fol. 206 ${ }^{\mathrm{r}}-212^{\mathrm{r}}$ vom 16.11. 1534 = AGAD, Perg. 3765); Nowy Sącz - ungen. ${ }^{32}$ Eine Art von Bestätigungen der Rechte und Berechtigungen waren auch die königlichen Urteile. Sie beendeten beispielsweise Streitigkeiten zwischen den Zünften. ${ }^{33}$

die Bestätigung der Jahrmärkte am 11. November (AGZ 9, 42 aus 1461); Osiek - die Bestätigung der Jahr- und Wochenmärkte (MK 36, S. 654-660 vom 6.5. 1524); Pierzchnica - die Bestätigung des Jahrund Wochenmarkts (MK 31, S. 27-28 vom 29. 12. 1517); Pilzno - die Bestätigung des Jahrmarkts (ANKr., Dok.Dep. 109 vom 22. 12. 1556); Radoszyce die Bestätigung des Jahr- und Wochenmarkts (MK 20, fol. 149 $-150^{\mathrm{r}}$ aus Januar 1504); Ryczywół - die Bestätigung des Wochenmarkts am Sonntag (ZDM 6, 1557 vom 12. 6. 1389); Solec (MK 33, S. 12-16 vom 5. 9. 1518); Stężyca - die Bestätigung der Jahr- und Wochenmarkten (MK 42, fol. 238v-239r vom 20. 5. 1527); Stopnica - die Bestätigung des Jahr- und Wochenmarkts (MK 38, S. 200-202 vom 17. 2. 1525); Wieliczka - die Bestätigung des Wochenmarkts (BJ, Perg. 51 vom 28. 8. 1447); Wojnicz - die Bestätigung der freien Fleischverkauf am Dienstag (ANKr., Dok.Dep. 166 vom 3.10.1521); Wolbrom - die Bestätigung des Wochenmarkts (MK 54, fol. 283r $-285^{\text {r }}$ vom 30. 12. 1537).

${ }^{32}$ Kęty - die Bestätigung der Hähnchentributbefreiung (MK 35, S. 290-291 vom 17. 8. 1521); Krakau - die Bestätigung der Steuerbefreiung (KDMK I.1, 132 aus 1435; KDMK I.1. 146 aus 1458; KDMK I.1, 187 aus 1475; PPiSMK I.1, 139 vom 4. 12. 1550); Krakau - die Bestätigung der Steuerbefreiung (sog. czopowe) für die Krakauer Schankwirten (PPiSMK I.2, 560 vom 23. 7. 1566); Krakau - Erneuerung der verbrannten Urkunden (PPiSMK I.2, 560 vom 23. 7. 1566); Łuków die Bestätigung der Steuersenkung (MK 49, fol. 206 ${ }^{\mathrm{r}}$ $212^{\mathrm{r}}$ vom 16.11. 1534); Oświęcim - die Bestätigung der Steuerbefreiung (MK 17, fol. 343 ${ }^{\mathrm{v}}-344^{\mathrm{r}}$ aus April 1502); Urzędów - die Bestätigung der Zinsenbefreiung (MK 22, fol. 107r-107v vom 17. 3. 1508); Żywiec - die Bestätigung der Steuerumtausch (MŻywiec 5 vom 7. 11. 1544).

${ }^{33}$ Beispielweise: Fleischer aus Krakau vs. Fleischer aus Kleparz (MK 25, fol. $24^{\mathrm{r}}$ aus 1511); Krakauer Schneider und Krakauer Gerbern (PPiSMK I.1, 332 vom 11.3.1524); Goldschmiede aus Kazimierz vs. Goldschmiede aus Krakau (PPiSMK I.1, 352 vom 11. 11. 1531; PPiSMK I.1, 433 vom 20.12. 1544); Bierbrauern aus Kazimierz vs. Brauern aus Kazimierz (PPiSMK I.1, 358 vom 19.4. 1532); Krakauer Gerber vs. andere Lederarbeiter (PPiSMK I.1, 357 vom 19. 4. 1532); Krakauer Stadtrat vs. Krakauer Zinngießer (PPiSMK I.1, 364 vom 19.11. 1532); 


\section{4.}

Königliche Garantien spielten eine wichtige Rolle besonders für jene Akte, die Verhältnisse zwischen der Stadt und äußeren Rechtsubjekten regulierten. Meistens wurden diese Probleme nicht im primären Recht geregelt, denn sie gehörten nur zur königlichen Kompetenz (Zölle, Steuern, wirtschaftliche Zulassungen). Anders war es bei Fragen, die schon früher im primären Recht geregelt wurden - beispielsweise im Bereich des Privatrechts und Strafrechts. Die Projekte der neuen Regulationen wurden von Stadträten bearbeitet. Sehr oft betrafen die Änderungen des Privatrechts das Erbrecht genaugenommen gerada und hergeweth, das Testament u.a. Krakau war diesbezüglich ein Vorläufer; schon im Jahre 1336 hat Kazimierz der Große dort die Erbrechtswillkür bestätigt.

Diese Willküre aus Krakau waren später ein Muster für andere Städte, unter anderen für Nowy Sacz und Biecz. ${ }^{34}$ Das Gesetzgebungsverfahren bestand in diesen Fällen aus fünf Elementen: 1.) der Stadtrat fragte bei einer anderen Stadt nach deren Erbrechtsregulation, 2.) diese Regulation wurde von dem Stadtnotar bearbeitet, 3.) später sollte dieser Entwurf von allen Bürgern während der allgemeinen Versammlung (burding) bestätigt werden (dies war am häufigsten der Fall), 4.) die obligatorische königliche Zustimmung, 5.) die Promulgation.

Im Falle des Privatrechts finden wir in den Quellen keinen Streit zwischen Städten und äußeren Rechtsubjekten (beispielweise andere Städte oder königlichen Beamten wie Starostei und Zollbeamter), deshalb brauchten die Städte keine Garantieakten. Wahrscheinlich wird deshalb auch in den Quellen erwähnt, dass sich der Stadtrat nicht immer sofort an den König wandte, um die obligatorische Zustimmung für

Fleischer aus Krakau vs. Fleischer aus Wojnicz (PPiSMK I.1, 482 vom 6. 5. 1552); Tuchfachmann aus Kazimierz vs. Magnus Procurator Cracoviensis (königlicher Beamter - PPiSMK I.1, 509 vom 9. 6. 1559).

${ }^{34}$ Nowy Sącz (AGZ 9, 11 vom 9.3. 1403); Biecz (ZDM 7, 1948 vom 22. 8. 1423; MBiecz, 29). das neue Recht zu bekommen. Beispielsweise bestätigte Zygmunt August (1548-1572) erst im Jahre 1565 die Erbrechtwillkür für die Stadt Kazimierz bei Krakau von 1548. ${ }^{35}$ Diese Willkür wurde also schon früher angewendet, was natürlich im Widerspruch zum Magdeburger Recht stand. Der Oberhof zu Burg zu Krakau hat schon im Jahre 1469 erklärt, dass die Zustimmung des Königs für die neue Regulation obligatorisch sei. Diese Entscheidung aus dem Jahre 1469 betraf auch das Erbrecht. Im Tenor lesen wir, dass die Frau die Rechte zu Gerada seu parapherna habe soweit eine andere Regulation vom König nicht eingeführt werde: ,[...] res ad parafernalia alias gratty spectant, et si tunc vestra civitas non habet aliqua specialia munimenta et privilegia super huiusmodi parafarnalia, donata et confirmata per Regiam Maiestatem, extunc res iste, que sunt parafarnalia, videlicet ornamenta muliebria [...] iure pociori ad Hedwigim spectare dinoscuntur. “" 36

\section{5.}

Die Modifikation und die Entwicklung des Stadtrechts in Polen ist ein Thema, das noch weiter intensive Forschungen braucht, unter anderem zu Fragen der Rechtsanwendung, Wirksamkeit und Gründung der Rechtsentwicklung. Auf dem Gebiet des Gesetzgebungsverfahrens sollte man besonders persönliche Abhängigkeiten klären, die Einfluss auf die Gesetzgebung hatten. Ohne Zweifel kann man sagen, dass die Rolle des Königs bei den Rechtsreformationen sehr groß war. Die Entstehung neuer legislativer Lösungen war von ihm wie auch von den obersten (aber begrenzten) Rechtsgebern abhängig. Der König war aber sehr oft nur theoretischer Rechtsgeber. Seine Entscheidungen basierten auf Informationen seiner Sekretäre und Kanzleien, deren Bedeutung immer grösser wurde. Wir können sehr oft nicht prüfen, inwieweit der König über bestimmte Sachen tatsächlich informiert war.

\footnotetext{
${ }^{35}$ PPiSMK I.1, 198 vom 5. 2. 1565.

${ }^{36}$ Decreta, 1037 aus 1469.
} 
Das zeigt, dass seit dem Ende des 15. Jhs die Rolle der zentralen Ämter und königlichen Beamten immer größer wurde. Die Bücher zur Polnischen Metrik bestätigen diese These.

\section{Korrespondenz:}

Maciej Mikuła

Jagiellonian University

Faculty of Law and Administration

Chair of Polish Legal History

Chair of Church Law and Law on Religious

Denominations

Gołębia St. 9, 31-007 Kraków, Poland

maciej.mikula@uj.edu.pl

\section{Abkürzungen:}

AGAD Archiwum Główne Akt Dawnych w Warszawie (Hauptarchiv für Altakten in Warszawa).

AGZ Onufry Pietruski, Xawery Liske (Hgg.), Akta grodzkie i ziemskie z czasów Rzeczypospolitej Polskiej z Archiwum tak zwanego bernardyńskiego we Lwowie wskutek fundacji śp. Aleksandra hr. Stadnickiego wydane staraniem Galicyjskiego Wydziału Krajowego, Bd. 4, 5, 9 (Lwów 1873-1883).

ANKr. Archiwum Narodowe w Krakowie (Nationalarchiv in Kraków).

BCzart. Biblioteka Czartoryskich w Krakowie (Bibliothek von Czartoryscy in Kraków).

BJ Biblioteka Jagiellońska (Jagiellonen Bibliothek).

Decreta Ludwik ŁYSIAK, Karin NEHLSEN-VON STRYK (Hgg.), Decreta iuris supremi Magdeburgensis castri Cracoviensis: Die Rechtssprüche des Oberhofs des deutschen Rechts auf der Burg zu Krakau, 1456-1481 (Frankfurt am Main 1995).

Dok.Dep. Depoturkunde in ANKr.

Heck Walerian HECK (Hg.), Archiwa miejskie księstw oświęcimskiego i Zatorskiego (Kraków 1891).

JIM Mikołaj JASKIER, Iuris Municipalis Maideburgensis Liber vulgo Weichbild nuncupatur [...] (Samosci 1602).
KDM Franciszek PIEKOsIŃSKI (Hg.), Kodeks dyplomatyczny Małopolski (Codex diplomaticus Minoris Poloniae), Bd. IV (Kraków 1905).

KDM 5 Materialen zum Codex diplomaticus Minoris Poloniae, Bd. 5, Depot in: Arbeitsstelle für das HistorischGeographische Lexikon Kleinpolens im Mittelalter, Polnische Akademie der Wissenschaft in Krakau.

KDKM Franciszek PIEKOsIŃSKI (Hg.), Kodeks dyplomatyczny miasta Krakowa, Bd. I/1 (Kraków 1879).

KDW Antoni Gąsiorowski, Tomasz Jasiński (Hgg.), Kodeks dyplomatyczny Wielkopolski (Codex diplomaticus civitatis Cracoviensis, 1257-1506), Bd. VIII-X (Warszawa-Poznań 1989-1993).

Łabęcki 2 Hieronim ŁABĘCKI, Górnictwo w Polsce. Opis kopalnictwa i hutnictwa polskiego pod względem technicznym, historyczno-statystycznym i prawnym, Bd. 2 (Warszawa 1841).

MAML Antoni ProchasKA (Hg.), Materyały archiwalne wyjęte głównie $\mathrm{z}$ metryki litewskiej od 1348 do 1607 roku (Lwów 1890).

MBiecz Franciszek BUJAK (Bearb.), Materiały do historii miasta Biecza (1361-1632) (Kraków 1914).

Mik.B. Maciej MiKUŁA, Beneficjenci indywidualnych zwolnień podatkowych $\mathrm{w}$ małopolskich miastach królewskich w dobie jagiellońskiej, in: Cracow Studies of Constitutional and Legal History 5/1 (2012) 13-25.

Mik.R. Maciej MikuŁA, O reformie prawa miejskiego w XVI wieku: ciężkowicka uchwała o prawie prywatnym i administracji, in: Cracow Studies of Constitutional and Legal History 6/3 (2013) - im Druck.

MK Metryka Koronna (Polnische Metrik in AGAD).

MLublin Jan RIABININ (Bearb.), Materiały do historii miasta Lublina 1317-1792 ([Lublin] 1938).

MŻywiec Franciszek LeNCZOWSKI (Hg.), Materiały do dziejów miasta Żywca od XV do XVIII wieku (Kraków 1957).

Perg. Pergamenturkunde.

PPiSMK Franciszek PIEKOsIŃSKI (Hg.), Prawa, przywileje i statuty miasta Krakowa 
(Leges, privilegia et statuta civitatis Cracoviensis 1507-1795), Bd. I/1 (Kraków 1885).

Prz.Kal. Witold MAISEL (Hg.), Przywileje Kalisza, in: Rocznik Kaliski 6 (1973) 263-362.

SzIM Paweł SzCZERBIC, Ius Municipale, to jest prawo miejskie majdeburskie nowo $\mathrm{z}$ łacińskiego i z niemieckiego na polski język z pilnością i wiernie przełożone, hg. von Grzegorz Maria KOWALSKI (Kraków 2011).

ZDM Stanisław KURAŚ, Irena SUŁKOWSKAKURAŚ (Hgg.), Zbiór dokumentów małopolskich, Bd. 2, 3, 4, 6, 7 (WrocławWarszawa-Kraków-Gdańsk 1964, 1969, 1970, 1974, 1975).

\section{Literatur:}

Juliusz BARDACH, Recepcja w historii państwa i prawa, in: Czasopismo Prawno-Historyczne 29 (1977) 1-26.

Maria BogUCKA, Jarmarki w Polsce w XVI-XVIII wieku, in: Studia nad dziejami miast 15-25.

Wieland CARLS, Katalin GÖNCZI, Forschungsüberblick, in: Inge BILY, Wieland CARLS, Katalin GÖNCZI, Sächsisch-magdeburgisches Recht in Polen. Untersuchungen zur Geschichte des Rechts und seiner Sprache (Berlin 2011) 39-67.

Wieland CARLS, Rechtsquellen Sächsisch-magdeburgischen Rechts, in: Inge BILY, Wieland CARLS, Katalin GÖNCZI, Sächsisch-magdeburgisches Recht. in Polen. Untersuchungen zur Geschichte des Rechts und seiner Sprache (Berlin 2011), 69109.

Krzysztof CHŁAPOWSKI, u.a. (Bearb.), Urzędnicy centralni i nadworni XIV-XVIII w. (Kórnik 1992).

Friedrich EBEL, Rechtsentstehung und Rechtstransfer im Spiegel der Überlieferung (Magdeburger und Lübecker Recht), in: Heiner LÜCK, Matthias PUHLE, Andreas RANFT (Hgg.), Grundlagen für ein neues Europa. Das Magdeburger und Lübecker Recht in Spätmittelalter und Früher Neuzeit (KölnWeimar-Wien 2009) 37-47.

Alfred HALBAN, Zur Geschichte des deutschen Rechtes in Podolien, Wolhynien und der Ukraine (Berlin 1896).

Katalin GÖNCZI, Geschichtlicher Überblick zum Untersuchungen unter besonderen Berücksichtigung der Rechtsentwicklung, in: Inge BILY, Wieland CARLS, Katalin GÖNCZI, Sächsischmagdeburgisches Recht in Polen. Untersuchungen zur Geschichte des Rechts und seiner Sprache [Ivs Saxonico-Maidebvrgense in Oriente 2] (Berlin/ Boston: De Gruyter 2011) 13-37.

Bartłomiej GROICKI, Artykuły prawa majdeburskiego (Kraków 1616).

Bartłomiej GROICKI, Porządek sądów y spraw miejskich prawa maydeburskiego w Koronie Polskiej (Kraków 1616)

Rudolf von IHERING, Der Zweck im Recht, Bd.1, (Lepizig ${ }^{31893) . ~}$

Danuta JANICKA, Die Rezeption des Sachsenspiegels und des Magdeburger Rechts am Beispiel von Thorn im Kulmer Land, in: Ernst EICHLER, Heiner LÜCK (Hgg.), Rechts- und Sprachtransfer in Mittelund Osteuropa: Sachsenspiegel und Magdeburger Recht; internationale und interdisziplinäre Konferenz in Leipzig vom 31. Oktober bis 2. November 2003 [Ivs Saxonico-Maidebvrgense in Oriente 1] (Berlin: De Gruyter Recht 2008) 61-74.

Krystyna JANICKA, Wkład polskich historyków prawa $\mathrm{w}$ badania nad prawem magdeburskim w XX w. (1945-2010), in: Studia Iuridica Toruniensia, 11 (2012) 46-75.

Krystyna KAMIŃSKA, Lokacje miast na prawie magdeburskim na ziemiach polskich do $1370 \mathrm{r}$. Studium historyczno-prawne (Torun 1990).

Rolf LIEBERWIRTH, Einführung oder Rezeption? Mittelalterlich deutsches Recht in slawischen Herrschaftsgebieten. Das Beispiel: Polen, in: Ernst EICHLER, Heiner LÜCK (Hgg.), Rechts- und Sprachtransfer in Mittel- und Osteuropa 167-180.

Michael LUDWIG, Besteuerung und Verpfändung königlicher Städte im spätmittelalterlichen Polen (Berlin 1984).

Heiner LÜCK, Sachsenspiegel und Magdeburger Recht. Europäische Dimensionen zweier mitteldeutscher Rechtsquellen (ADIUVAT in Itinere; 5, Hamburg 1998).

Heiner LÜCK, Über den Sachsenspiegel. Entstehung, Inhalt und Wirkung des Rechtsbuches (2. Auflage, Dößel Saalekreis 2005).

Heiner LÜCK, Sachsenspiegel und Magdeburger Recht. Grundlagen für Europa. Eine Ausstellung des Landes Sachsen-Anhalt, in: Denkströme. Journal der Sächsischen Akademie der Wissenschaften (4/2010) 81-104.

Ludwik ŁYSIAK, Ius supremum Maydeburgese castri Cracoviensis 1356-1794. Organisation, Tätigkeit und Stellung des Krakauer Oberhofs in der Rechtsprechung Altpolens (Klostermann, Frankfurt am Main 1990).

Jacek S. MATUSZEWSKI, Tadeusz SzULC Opodatkowanie i polityka zastawu miast królewskich $\mathrm{w}$ 
Polsce za Jagiellonów. Uwagi w związku z książką Michaela Ludwiga „Besteuerung und Verpfändung königlicher Städte im Spätmittelalterlichen Polen", in: Czasopismo PrawnoHistoryczne 61/2 (1989) 175-192.

Józef MATUSzEWSKI, Prawo sądowe na wsi polskiej lokowanej na prawie niemieckim, in: Józef MAtuszewsKi (Hg.) Studia z Dziejów Państwa i Prawa Polskiego, Bd. 2 (Łódź 1995) 40-75.

Józef MATUSZEWSKI, Rodzaje własności gruntu we wsi lokowanej na prawie niemieckim, in: Katarzyna IWANICKA, Maria SKOWRONEK, Kazimierz STEMBrowicz (Hgg.), Parlament, prawo, ludzie. Studia ofiarowane Profesorowi Juliuszowi Bardachowi (Warszawa 1996) 158-164.

Józef MATUSZEWSKI, Prawo niemieckie w Statutach Kazimierza Wielkiego, in: Jacek S. MATUSZEWSKI (Hg.), Studia z Dziejów Państwa i Prawa Polskiego, Bd. 3 (Łódź 1999) 53-80.

Heinz MohnHAupt, Confirmatio privilegiorum, in: B. Dölemeyer, H. Mohnhaupt (Hgg.), Das Privileg im europäischen Vergleich, Bd. 2 (Vittorio Klostermann: Frankfurt am Main 1999) 45-63.

Zdzisław NoGA, Krakowska rada miejska w XVI wieku. Studium o elicie władzy (Kraków 2003).

Zdzisław NoGA, Relacje władz miejskich Krakowa z dworem i urzędnikami państwowymi w wieku XVI, in: Ryszard Skowron (Hg.), Dwór a kraj. Między centrum a peryferiami władzy. Materiały konferencji naukowej na Wawelu zorganizowanej przez Zamek Królewski na Wawelu, Instytut Historii UJ, Instytut Historii Akademii Pedagogicznej w Krakowie w dn. 2-5 kwietnia 2001, (Kraków 2003) 119-135.

Anna OdRZYWOLSKA-KIDAWA, Prace kancelaryjne podkanclerzego Piotra Tomickiego 1515-1535, in: Waldemar CHORAzŻYCZEWSKI, Wojciech KRAWCZUK, (Hgg.), Polska kancelaria królewska czasów nowożytnych. Między władzą a społeczeństwem, Teil 2 (Kraków 2006) 157-177.

Ulrich-Dieter OpPITZ, Deutsche Rechtsbücher des Mittelalters, Bd. 1 (Köln-Wien 1990).

Ilpo Taipani PIIRAINEN, Winfried WASSEN (Hgg.), Der Sachsenspiegel aus Oppeln und Krakau (Berlin 1996).

Zygfryd RYMASZEWSKI, Łacińskie teksty Landrechtu Zwierciadła Saskiego w Polsce. Versio Wratislaviensis - versio Sandomiriensis - Łaski (Warszawa 1985).
Zygfryd RYMASZEWSKI, Król w dawnej Polsce w świetle dokumentów praktyki sądowej, in: Jacek S. MatuszewsKi, Tadeusz KubICKI (Hgg.), Studia z Dziejów Państwa i Prawa Polskiego 7 (2002) 173181.

Sachsenspiegel; Weichbildrecht, Das Sächsische; Ius municipale Magdeburgense Versio Sandomiriensis; Ius municipale Magdeburgense Versio Nicolai Iaskier, in: Geschichtsquellen des deutschen Mittelalters [www.geschichtsquellen. de] (abgerufen am: 29. 10. 2013).

Henryk SAMSONOWICZ, Handel Lublina na przełomie XV i XVI wieku, in: Przegląd Historyczny 59 (1968) 612-628.

Henryk SAMSONOWICZ, Przemiany osi drożnych w Polsce późnego średniowiecza, in: Przegląd Historyczny 64 (1973) 697-716.

Henryk SAMSONOWICZ, Niektóre cechy charakterystyczne sieci jarmarcznej w Polsce późnego średniowiecza, in: Studia nad dziejami miast i mieszczaństwa w średniowieczu, Bd. 1 (Toruń 1996) 9-14.

Henryk SAMSONOwicz, Kto podejmował decyzje w miastach samorządowych średniowiecznej Polski? in: Zenon PIECH (Hg.), Miasta, ludzie, instytucje, znaki. Księga jubileuszowa ofiarowana Profesor Bożenie Wyrozumskiej w 75. rocznicę urodzin (Kraków 2008) 149-159.

Marcin STARZYŃSKI, Krakowska rada miejska w średniowieczu (Kraków 2010).

Irena SUŁKOWSKA-KURAŚ, Dokumenty królewskie i ich funkcja w państwie polskim za Andegawenów i pierwszych Jagiellonów (Warszawa 1977).

Andrzej WYROBISZ, Uwagi o kalendarzu jarmarków, in: Studia nad dziejami miast 27-32.

Stanisław Marian ZAJĄCZKOWSKI, Lokacje osad wiejskich na prawie niemieckim na obszarze przedrozbiorowego powiatu łęczyckiego do lat siedemdziesiątych XVI wieku (Łódź 2003).

Aleksander ZAJDA, Deutsche Einflüsse in der altpolnischen juristischen Terminologie als Widerspiegelung der Rezeption des Magdeburger Rechts, in: Ernst EICHLER, Heiner LÜCK (Hgg.), Rechts- und Sprachtransfer in Mittel- und Osteuropa 289-304.

Maciej ZDANEK (Hg.), Kopiariusz cystersów szczyrzyckich z XVII wieku w zbiorach Biblioteki Naukowej PAU i PAN, in: Rocznik Biblioteki Naukowej PAU i PAN w Krakowie 54 (2009) 2352. 